\title{
Effectiveness of Traditional Chinese Medicine Compound JieDuTongLuoShengJin Granules Treatment in Primary Sjögren's Syndrome: A Randomized, Double-Blind, Placebo-Controlled Clinical Trial
}

\author{
Ben Li, ${ }^{1}$ Jiaqi Hou, ${ }^{1}$ Yue Yang, ${ }^{1}$ Xuemei Piao, ${ }^{1}$ Yueying Chen, ${ }^{2}$ Luan Xue, ${ }^{1}$ Dan Wang, \\ Jiandong Hu, ${ }^{1}$ Guoling Li, ${ }^{1}$ Xiangxiang $\mathrm{Wu}^{1}{ }^{1}$ Yu Sun, ${ }^{1}$ and Runa $\mathrm{Jin}^{3}$ \\ ${ }^{1}$ Department of Rheumatology and Immunology, Yueyang Hospital of Integrated Traditional Chinese and Western Medicine, \\ Shanghai University of Traditional Chinese Medicine, Shanghai 200437, China \\ ${ }^{2}$ Shanghai University of Traditional Chinese Medicine, Shanghai 200437, China \\ ${ }^{3}$ Department of Ophthalmology, Yueyang Hospital of Integrated Traditional Chinese and Western Medicine, \\ Shanghai University of Traditional Chinese Medicine, Shanghai 200437, China \\ Correspondence should be addressed to Luan Xue; xelco@163.com
}

Received 27 February 2017; Revised 5 May 2017; Accepted 5 June 2017; Published 10 July 2017

Academic Editor: Lesley Ward

Copyright (C) 2017 Ben Li et al. This is an open access article distributed under the Creative Commons Attribution License, which permits unrestricted use, distribution, and reproduction in any medium, provided the original work is properly cited.

Objective. To evaluate the clinical therapeutic efficacy and safety of JieDuTongLuoShengJin granules + HCQ in patients with pSS Methods. 40 patients with low-activity-level pSS and without visceral involvement participated in this study and were randomized to receive either JieDuTongLuoShengJin granules with HCQ or placebo with HCQ. Patients and investigators were blinded to treatment allocation. The primary endpoint was week 12 ESSPRI score, while secondary endpoints included ESSDAI, salivary and lacrimal gland function, and some laboratory variables. Safety-related data were also assessed. Results. Comparing with the placebo group, the treatment group experienced statistically significant improvement in the mean change from baseline for the primary endpoint of ESSPRI score and also in PGA. Moreover, in comparison with baseline values, the treatment group had significantly improved ESSDAI score, unstimulated saliva flow rate, and several laboratory variables. However, upon comparison of the two groups, there were no significant differences for them. The incidence of AEs was 10.0\%, one in treatment group and three in placebo group. Conclusion. Treatment with a combination of JieDuTongLuoShengJin granules with HCQ is effective in improving patients' subjective symptoms and some objective indicators of pSS. These results indicate that JieDuTongLuoShengJin is promising as a safe and effective treatment of pSS.

\section{Introduction}

Sjögren's syndrome is a systemic diffuse connective tissue disease that is characterized by chronic, autoimmune inflammation of exocrine glands, especially the salivary and lacrimal glands, resulting in glandular hypofunction. In addition to xerostomia and keratoconjunctivitis sicca, Sjögren's syndrome may frequently be accompanied by arthralgia and myalgia and $85 \%$ of patients experience severe fatigue. Furthermore, some patients have other serious extraglandular involvement-such as the kidneys, liver, and lungs-and manifest corresponding symptoms [1]. Laboratory manifestations, such as the presence of anti-SSA and anti-SSB autoantibodies and increased serum levels of rheumatoid factor (RF), are due to B cell hyperactivity.

Primary Sjögren's syndrome (pSS) has developed into one of the most common autoimmune rheumatic diseases in the USA, affecting three million people [2]. The prevalence in China is about $0.3 \%$ to $0.4 \%$, second only to that of rheumatoid arthritis (RA) [3]. The incidence of Sjögren's syndrome in the elderly is estimated to be 3\%-4\% [4].

Sjögren's syndrome can have significant impacts on patients' health-related quality of life, leading to many social problems. However, to date there is no curative systemic 
treatment for pSS. The main goal of treatment is to relieve clinical symptoms, prevent further organ damage due to disease progression, and prolong patients' survival. In recent years, there has been extensive study of new treatment methods for Sjögren's syndrome, including rituximab, belimumab, and others. However, most have failed to show significant improvement in patients' important symptoms such as dryness, fatigue, and pain [5-7]. For those Sjögren's Syndrome patients without visceral involvement, the most common treatments are moisture replacement therapies and immunosuppressants such as especially hydroxychloroquine (HCQ) and prednisone [8]. Treatment choice is based on the physicians' clinical experience, expert opinion, and limited clinical research $[9,10]$. Chinese herbal medicine $(\mathrm{CHM})$ is widely used in the treatment of pSS in China and improves patients' subjective symptoms in some clinical research [11, 12]. Sjögren's syndrome belongs to the "dryness arthralgia" category in traditional Chinese medicine. Following the pathogenesis of "deficiency, stasis, and toxicity," we set up JieDuTongLuoShengJin, which is a recipe that has been felt to be effective in the bedside treatment of pSS. This study contributes to the ongoing research in effective, evidencebased interventions for Sjögren's syndrome.

\section{Patients and Methods}

2.1. Patient Selection. All pSS patients admitted to Yueyang Hospital of Integrated Traditional Chinese and Western Medicine affiliated with Shanghai University of Traditional Chinese Medicine between August 2014 and February 2016 were potential study candidates. All patients were between 18 and 70 years of age and fulfilled the American European Consensus Group criteria for pSS [13]. Eligibility criteria were ESSDAI score of $<5$, being without visceral involvement, and belonging to Yin deficiency on the basis of the traditional Chinese medicine. In addition, results from a salivary gland biopsy performed within 12 months before inclusion and showing the characteristic features of SS had to be available. Patients were naive to prior treatment with disease-modifying antirheumatic drugs (DMARDs), or if they had had previous treatment, then treatment of those treated with hydroxychloroquine or prednisone had to be discontinued at least 1 month before baseline, and treatment of those treated with other DMARDs had to be discontinued at least 6 months before baseline. Patients were permitted to use artificial tears and saliva, but the regimen had to remain identical during follow-up, and they had to be stopped at least 1 day prior to each assessment. Patients with a history of any malignancy or with underlying cardiac, cerebrovascular, pulmonary, liver, renal, or hematopoietic system conditions, chronic or latent infectious diseases (e.g., Human Immunodeficiency Virus and Acquired Immune Deficiency Syndrome (HIV/AIDS) and hepatitis C virus (HCV) infection), immune deficiency, and macular degeneration and pregnant women were excluded. During the study, patients who had been in other clinical trials were excluded. For patients who had emerging extraglandular manifestations, those who developed these within four weeks of study initiation were removed from the study; after four weeks they were terminated early from the study but their data kept for analysis. All patients underwent laboratory assessment, electrocardiography, and chest radiography at baseline.

2.2. Study Design. The study was conducted according to the Declaration of Helsinki. The protocol was reviewed and approved by the institutional review board and ethics committee of Yueyang Hospital of Integrated Traditional Chinese and Western Medicine affiliated with Shanghai University of Traditional Chinese Medicine. And it was registered in Chinese Clinical Trial Registry (registration ID: ChiCTR-IPR-14005441). All patients provided their written informed consent. According to literature review data, the combination of traditional Chinese and western medicine treatment efficacy rate is about $80 \%\left(p_{1}\right)$, and that for pure western medicine treatment is $30 \%\left(p_{2}\right)$, and $\alpha=0.05$ and $\beta=0.10$. We formed the JieDuTongLuoShengJin granule group and placebo group in a ratio of $1: 1$. Using the formula $n=\left(z_{\alpha}+z_{\beta}\right)^{2} 2 p(1-p) /\left(p_{1}-p_{2}\right)^{2}$, where $n$ is the number of each group, $p=\left(p_{1}+p_{2}\right) / 2$. We calculated that the required number of cases was 34 . Taking into account the lost cases, 40 cases were designated for inclusion in this trial. 40 patients enrolled in this prospective, single-center, randomized, double-blind, placebo-controlled study were randomly divided into treatment group and control group $(1: 1)$ by SPSS 19.0 statistical software packages. The random coding table was stored in the clinical research database. A designated person was responsible for distributing the oral granules in accordance with the eligible patients' visiting sequence and test drug coding. All investigators and patients were blinded to treatment allocation. The dosage of placebo was a 1/10 dose of the JieDuTongLuoShengJin granules received by the treatment group. All patients in both treatment and placebo groups also received hydroxychloroquine (HCQ) $0.2 \mathrm{mg}$ by mouth twice daily. Data for clinical efficacy and safety followup based on physical examination and laboratory tests were collected at week 12. In addition, AEs and serious AEs were evaluated. An adverse event (AE) is any adverse change in health or "side-effect" including any symptoms, syndromes, or diseases observed in a patient during the clinical trial.

\subsection{Outcome Parameters}

2.3.1. Definition of Endpoints. The primary endpoint was defined as a significant improvement in the ESSPRI score in the JieDuTongLuoShengJin granules group compared with the placebo group. Secondary endpoints were an improvement in the ESSDAI score and measurement of salivary and lacrimal gland function. Laboratory variables were also measured. All variables were assessed at baseline (within 2 weeks before treatment) and at 4 and 12 weeks after treatment.

2.3.2. Evaluation of Disease Activity. The European League Against Rheumatism (EULAR) developed both consensus disease activity indexes used in this study: EULAR Sjögren's Syndrome Patient-Reported Index (ESSPRI) [14] and EULAR Sjögren's Syndrome Disease Activity Index (ESSDAI) [15]. 
The ESSPRI is a validated tool for subjective self-assessment of pSS disease activity. It contains three components: dryness, fatigue, and pain (joint and/or muscle pain), with a single 0-10 numerical scale for each of the three domains to assess patients' symptoms. The final score is obtained by averaging the scores of the three scales: (dryness + fatigue + pain)/3. The final ESSPRI score ranges from 0 to 10 . The patient-acceptable symptom state (PASS) estimate has been defined as an ESSPRI $<5$ points and the minimal clinically important improvement (MCII) as a decrease of at least one point or $15 \%$ [16].

The ESSDAI is a validated tool for global assessment of pSS disease activity by experienced clinicians. It includes 12 domains: constitutional, lymphadenopathy, glandular, articular, cutaneous, pulmonary, renal, muscular, peripheral nervous system, central nervous system, hematological, and biological domains. For each domain, features of disease activity are classified into three to four levels $(0=$ "no"; $1=$ "low"; 2 = "moderate"; 3 = "high") according to their severity. Each domain has a weight that ranges from 1 to 6 . The final score is obtained by multiplying the disease domain by the domain weight and then summing the scores of all domains. ESSDAI ranges from 0 to 123 . The MCII is defined as a decrease of at least three points. The EDDSAI is divided into three levels: low activity (ESSDAI $<5)$, moderate activity $(5 \leq$ ESSDAI $<14$ ), and high activity (ESSDAI $\geq 14)[16]$.

The ESSPRI score was completed for each patient at baseline, at week 4, and at study completion. The ESSDAI scores were completed for each patient at baseline and at study completion. In addition, patients completed a Patient Global Assessment (PGA), which evaluates the global severity of their symptoms related to their pSS using a 0-10 numerical scale. All questionnaires were conducted through in-person interviews due to participants' low education level.

2.3.3. Evaluation of Salivary and Lacrimal Gland Function. Unstimulated saliva can reflect salivary gland function. We collected unstimulated saliva using Lashley cups. Salivary volume was measured over 15 minutes between 1:00 and 4:00 $\mathrm{PM}$ on the day of assessment in order to minimize fluctuations related to the circadian rhythm of salivary secretion [1719].

Three tests were used to evaluate lacrimal gland function: Schirmer's test I, breakup time (BUT), and the van Bijsterveld Ocular Dye Score [20, 21]. Schirmer's test I was carried out by placing a tear test filter paper in the lower fornix of the conjunctiva of the eye. The length of wetting was measured after 5 minutes. The BUT is the interval between the last blink and the appearance of the first randomly distributed dry spots. It is assessed by instilling a $1 \%$ fluorescein solution in the fornix of both eyes. The BUT was checked by the same physician for all patients. The van Bijsterveld Ocular Dye Score was calculated by the same ophthalmologist for all patients, too. The lissamine green test was performed by instilling $1 \%$ lissamine green in both eyes. After 1 or 2 full blinks, black dots appeared in the ocular surface, which were observated by cobalt blue with a slit lamp microscope. The intensity of staining of both cornea and bulbar conjunctiva was scored. The ocular surface staining score was rated from
0 to 9 (up to 3 points for each section $[1=$ sparsely scattered, $2=$ densely scattered, and $3=$ confluent]) $[22,23]$.

2.3.4. Laboratory Assessment. Levels of immune globulin G (IgG), rheumatoid factor (RF), and C-reactive protein (CRP) were measured using rate nephelometry. The erythrocyte sedimentation rate (ESR) was measured with the Westergren method. Antinuclear antibodies (ANAs) were measured by indirect immunofluorescence and considered positive at a titer 1:80. Antibodies to SSA and SSB cellular antigens were determined using their enzyme linked immunosorbent assay (ELISA) kits.

2.4. Statistical Analysis. All statistical analyses involved use of SPSS 19.0 statistical software packages. The Shapiro-Wilk test was used to determine normality of continuous variables. Categorical data was summarized using counts/percentages. For continuous variables, those with normal distribution were analysed using the $t$-test and presented as "means \pm standard deviation." Those variables with abnormal distribution were analysed using the Mann-Whitney $U$ test and presented as "median (interquartile range)." Categorical variables were analysed using the chi-square test or Fisher's exact probability test. Analysis of covariance (ANCOVA) and logistic regression analysis were used for analysis of postintervention group differences. In the intent-to-treat analyses, a last observation carried forward (LOCF) procedure was employed to impute missing clinical efficacy data (e.g., ESSPRI, ESSDAI, or PGA) at week 12. Only two-sided tests were applied. All efficacy analyses were based on the intention-to-treat principle, and, for all statistical analyses, a $p$ value $<0.05$ was considered statistically significant.

\section{Results}

3.1. Patient Characteristics. 40 patients were recruited and randomly assigned to this randomized, double-blind, placebo-controlled study (Figure 1). All patients were followed for 12 weeks, with the first exam performed on 26 August 2014 and the last week 12 evaluation completed on 15 May 2016.

Patients' baseline characteristics were generally comparable between the two groups (Table 1). We found no differences in the characteristics such as sex, age, and clinical features. 20 patients who took JieDuTongLuoShengJin granules orally were identified with an average age of $51.6 \pm 11.28$ years (versus $52.3 \pm 12.40$ years in the placebo group) and an average disease duration of $5.6 \pm 5.12$ years (versus $5.6 \pm$ 3.65 years in the placebo group), and women constituted all of the population (versus $95.0 \%$ of the placebo group). There were no statistically significant differences in any pSS characteristics between the two groups $(p>0.05)$.

\subsection{Clinical Efficacy}

3.2.1. Disease Activity. After treatment, the between-group and within-group before-and-after paired comparison results were analysed. The ESSPRI (the primary endpoint) (Tables 2 and 3) significantly improved in both groups $(p=0.009$ at week 4 and $p=0.000$ at week 12 in the treatment 


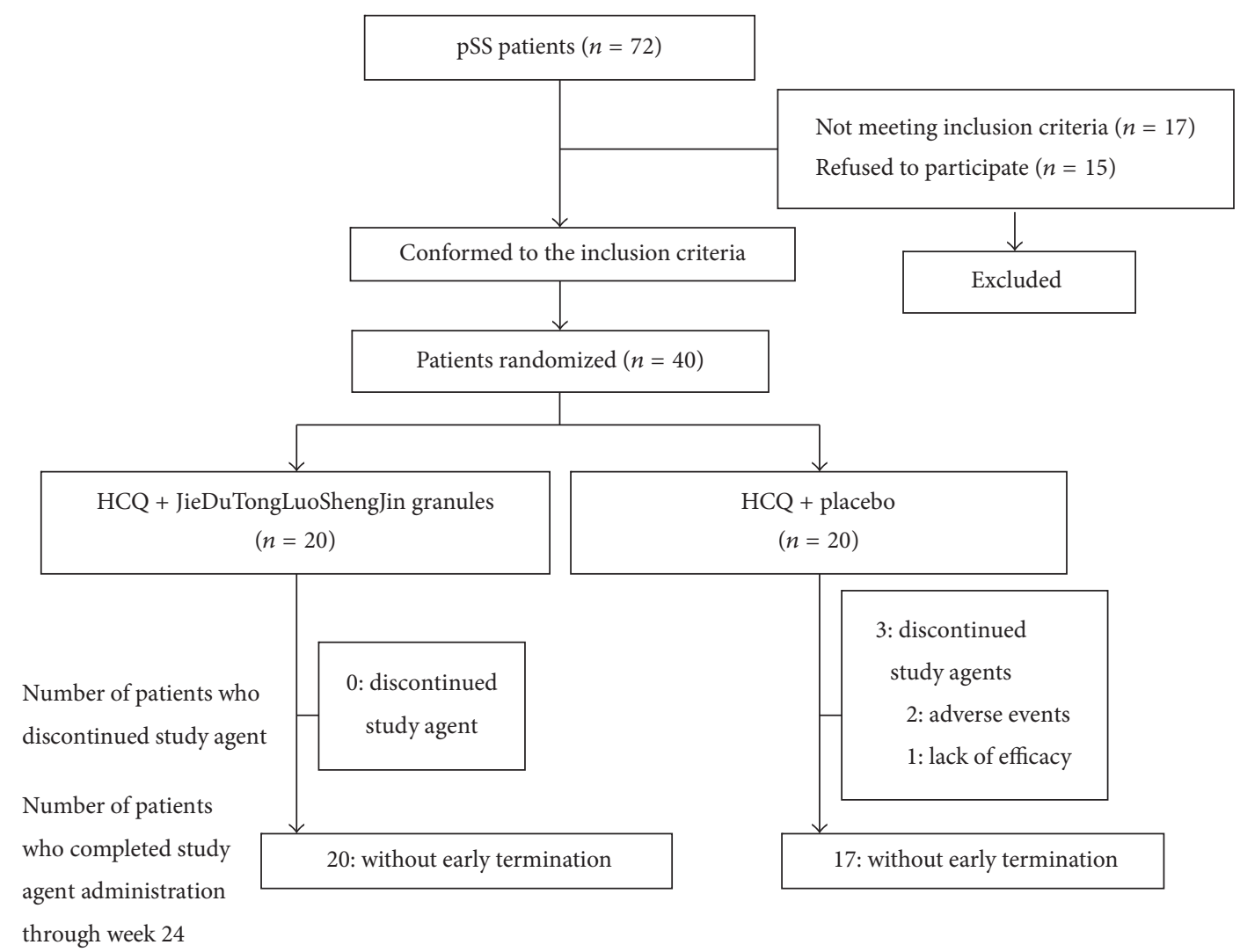

FIGURE 1: Patient disposition through week 12 of the trial. Randomization of patients with pSS into the two groups. Of a cohort of 183 patients, 40 patients were randomized into two treatment groups. HCQ: hydroxychloroquine.

group, $p=0.039$ at week 4 , and $p=0.032$ at week 12 in the placebo group, versus baseline), and a statistically significantly lower ESSPRI score was found in the JieDuTongLuoShengJin granules + HCQ group at week 12 $(p=0.002)$. Similar pattern of improvement was also observed for PGA (Table 3). The proportion of patients in the JieDuTongLuoShengJin granules + HCQ group achieving PASS increased from $45.00 \%$ at week 0 to $95.00 \%$ at week 12 $(p=0.001)$ and was significantly greater than that of the placebo + HCQ group (60.00\%) (Table 2). The percentage of those in the JieDuTongLuoShengJin granules + HCQ group who achieved MCII (85.00\%) was also significantly greater than that in the placebo + HCQ group (50.00\%) at week 12 (Table 2).

Compared to week 0 , assessments of each domain of ESSPRI also indicated significant improvement with JieDuTongLuoShengJin granules + HCQ at week $4(p=0.049$, $p=0.042$, and $p=0.034$, resp.) and at week $12(p=0.011$, $p=0.000$, and $p=0.006$, resp.). Patients in the placebo + HCQ group demonstrated significantly greater improvement in the pain domain $(p=0.025$ at week 4 and $p=0.014$ at week 12), but not dryness domain and fatigue domain. There was no statistical difference between the two groups in each domain of ESSPRI at week $4(p>0.05)$. At week 12 , patients in the JieDuTongLuoShengJin granules + HCQ group demonstrated a statistically significant reduction in the dryness score and fatigue score versus placebo + HCQ ( $p=0.026$ and $p=0.002$, resp.), but the differences for the pain score were not significant $(p>0.05)$ (Table 3$)$.

The ESSDAI score was one of the secondary endpoints. All of the patients showed low disease activity without organ involvement at the time of week 0 . The ESSDAI significantly improved in both groups at week 12 ( $p<0.01$ in the treatment group and $p<0.05$ in the placebo group, versus baseline). But no significant difference was found between the two groups $(p>0.05)$ (Table 3), and none of the patients achieved MCII (Table 2).

3.2.2. Salivary and Lacrimal Gland Function. At week 4, the unstimulated whole salivary flow rate increased to a certain extent in the treatment group, but there was no statistical difference $(p>0.05)$, and did not improve significantly in the control group (Table 3 ). The unstimulated whole salivary flow rate significantly increased from baseline in the treatment group $(p=0.000)$ more than in the placebo group $(p=$ 0.049 ) at week 12; however, this difference was not significant between the two groups at week $12(p>0.05)$ (Table 3$)$.

The between-group and within-group before-and-after paired comparison results of Schirmer's test, BUT test, and Corneal and Conjunctival staining were analysed and showed no significant changes in lacrimal gland function in either groups $(p>0.05)$ (Table 3$)$. 
TABLE 1: Summary of demographics and characteristics at baseline of pSS patients.

\begin{tabular}{|c|c|c|c|}
\hline & JieDuTongLuoShengJin granules + HCQ & Placebo + HCQ & $p$ value \\
\hline Patients randomized, $n$ & 20 & 20 & \\
\hline Age (years) & & & 0.857 \\
\hline Mean \pm SD & $51.6 \pm 11.28$ & $52.3 \pm 12.40$ & \\
\hline Median & 52 & 53 & \\
\hline Range & $(27,69)$ & $(24,68)$ & \\
\hline Gender, $n(\%)$ & & & 1.000 \\
\hline Male & $0(0.00)$ & $1(5.00)$ & \\
\hline Female & $20(100.00)$ & $19(95.00)$ & \\
\hline Disease duration (years) & & & 0.989 \\
\hline Mean \pm SD & $5.6 \pm 5.12$ & $5.6 \pm 3.65$ & \\
\hline Median (IQR) & $4.5(2,8.25)$ & $5(3,7.25)$ & \\
\hline Range & $(0.2,20)$ & $(0.5,15)$ & \\
\hline Dryness $(0-10)$ & & & 0.907 \\
\hline Mean \pm SD & $5.7 \pm 2.81$ & $5.8 \pm 2.55$ & \\
\hline Median (IQR) & $5.5(3,7.25)$ & $5.5(4.75,8)$ & \\
\hline Fatigue $(0-10)$ & & & 0.957 \\
\hline Mean \pm SD & $5.6 \pm 3.22$ & $5.7 \pm 2.64$ & \\
\hline Median (IQR) & $5(3.75,8.25)$ & $5(4,7.25)$ & \\
\hline Pain $(0-10)$ & & & 0.678 \\
\hline Mean \pm SD & $2.7 \pm 2.25$ & $2.4 \pm 2.50$ & \\
\hline Median (IQR) & $3(0,4)$ & $2(0,4)$ & \\
\hline ESSPRI (0-10) & & & 0.939 \\
\hline Mean \pm SD & $4.7 \pm 2.09$ & $4.6 \pm 1.99$ & \\
\hline Median (IQR) & $5(2.7,6.4)$ & $4.3(3.8,5.9)$ & \\
\hline PASS, $n(\%)$ & $9(45.00)$ & $11(55.00)$ & 0.752 \\
\hline PGA $(0-10)$ & & & 0.624 \\
\hline Mean \pm SD & $5.4 \pm 2.19$ & $5.8 \pm 2.29$ & \\
\hline Median (IQR) & $6(3,7)$ & $6(4,7)$ & \\
\hline ESSDAI (0-124) & & & 0.655 \\
\hline Mean \pm SD & $2.6 \pm 1.31$ & $2.4 \pm 1.31$ & \\
\hline Median (IQR) & $3(1,4)$ & $2(1,4)$ & \\
\hline Salivary flow rate $(\mathrm{ml} / 15 \mathrm{~min})$ & & & 0.989 \\
\hline Mean \pm SD & $0.8 \pm 0.70$ & $0.7 \pm 0.62$ & \\
\hline Median (IQR) & $0.8(0.1,1.0)$ & $0.7(0.2,1.0)$ & \\
\hline Schirmer's test (left) $(\mathrm{mm} / 5 \mathrm{~min})$ & & & 0.224 \\
\hline Mean \pm SD & $4.5 \pm 3.82$ & $4.0 \pm 4.66$ & \\
\hline Median (IQR) & $3.8(2.0,5.3)$ & $2.0(1.0,4.5)$ & \\
\hline Schirmer's test (right) $(\mathrm{mm} / 5 \mathrm{~min})$ & & & 0.126 \\
\hline Mean \pm SD & $4.3 \pm 3.01$ & $3.2 \pm 3.31$ & \\
\hline Median (IQR) & $4.0(2.0,6.3)$ & $2.0(1.0,4.0)$ & \\
\hline BUT (Left) (second) & & & 0.140 \\
\hline Mean \pm SD & $3.6 \pm 1.54$ & $3.0 \pm 1.69$ & \\
\hline Median (IQR) & $3.0(3.0,4.0)$ & $2.5(2.0,4.0)$ & \\
\hline BUT (Right) (second) & & & 0.633 \\
\hline Mean \pm SD & $3.4 \pm 1.57$ & $3.2 \pm 1.39$ & \\
\hline Median (IQR) & $3.0(2.8,3.3)$ & $3.0(2.0,4.0)$ & \\
\hline van Bijsterveld score (Left) & & & 0.622 \\
\hline Mean \pm SD & $3.5 \pm 1.88$ & $3.2 \pm 1.94$ & \\
\hline Median (IQR) & $4.0(2.0,5.0)$ & $3.0(2.0,4.0)$ & \\
\hline van Bijsterveld score (Right) & & & 0.695 \\
\hline Mean \pm SD & $3.0 \pm 1.69$ & $3.2 \pm 1.51$ & \\
\hline Median (IQR) & $3.0(2.0,4.0)$ & $3.0(2.8,4.0)$ & \\
\hline
\end{tabular}


TABle 1: Continued.

\begin{tabular}{|c|c|c|c|}
\hline & JieDuTongLuoShengJin granules + HCQ & Placebo + HCQ & $p$ value \\
\hline $\operatorname{IgG}(\mathrm{g} / \mathrm{L})$ & & & 0.596 \\
\hline Mean \pm SD & $18.3 \pm 3.07$ & $19.2 \pm 6.91$ & \\
\hline Median (IQR) & $17.8(16.4,20.8)$ & $17.3(14.3,20.5)$ & \\
\hline $\mathrm{C} 3(\mathrm{~g} / \mathrm{L})$ & & & 0.204 \\
\hline Mean \pm SD & $0.9 \pm 0.20$ & $0.8 \pm 0.20$ & \\
\hline Median (IQR) & $0.9(0.8,1.0)$ & $0.9(0.8,1.0)$ & \\
\hline $\mathrm{C} 4(\mathrm{~g} / \mathrm{L})$ & & & 0.071 \\
\hline Mean \pm SD & $0.2 \pm 0.05$ & $0.2 \pm 0.05$ & \\
\hline Median (IQR) & $0.2(0.2,0.2)$ & $0.2(0.1,0.2)$ & \\
\hline $\operatorname{ESR}(\mathrm{mm} / \mathrm{h})$ & & & 0.871 \\
\hline Mean \pm SD & $35.9 \pm 25.74$ & $34.4 \pm 31.83$ & \\
\hline Median (IQR) & $36.5(14.3,46.3)$ & $23.5(18.0,40.3)$ & \\
\hline $\mathrm{CRP}(\mathrm{mg} / \mathrm{L})$ & & & 0.756 \\
\hline Mean \pm SD & $3.4 \pm 2.69$ & $3.4 \pm 2.81$ & \\
\hline Median (IQR) & $2.4(2.1,3.2)$ & $2.6(1.7,4.2)$ & \\
\hline RF positive, $n(\%)$ & $9(45.0)$ & $7(35.0)$ & 0.748 \\
\hline \multicolumn{4}{|l|}{ Autoantibodies } \\
\hline Anti-SSA & $14(70.0)$ & $16(80.0)$ & 0.716 \\
\hline Anti-SSB & $4(20.0)$ & $3(15.0)$ & 1.000 \\
\hline
\end{tabular}

TABLE 2: Comparison of clinical curative effect in the two groups after treatment at week $12(n(\%))$.

\begin{tabular}{cccc}
\hline & JieDuTongLuoShengJin granules + HCQ & Placebo + HCQ & $\begin{array}{c}p \text { value } \\
\text { (between-group) }\end{array}$ \\
\hline PASS, $n(\%)$ & $19(95.00)$ & $12(60.00)$ & 0.024 \\
ESSPRI & & & \\
MCII, $n(\%)$ & $17(85.00)$ & $10(50.00)$ & 0.024 \\
ESSPRI & $0(0.00)$ & $0(0.00)$ & - \\
ESSDAI & & & - \\
\hline
\end{tabular}

3.2.3. Laboratory Assessment. ESR level was significantly lower from baseline after treatment in both groups, but not for the levels of serum complement (C3 and C4) as well as CRP. Serum IgG lever improved significantly from baseline in patients treated with JieDuTongLuoShengJin granules ( $p=$ $0.010)$, but not in patients treated with placebo $(p=0.096)$. All laboratory variables mentioned above had no statistical differences between the two groups after treatment at week 12 (Table 3).

3.2.4. Safety. During the study, adverse event happened in four patients. Similar proportions of patients from the treatment and placebo groups reported adverse event through week $4(5.0 \%$ and $0.0 \%$, resp.; $p>0.05)$ and week $12(5.0 \%$ and $15.0 \%$, resp.; $p>0.05$ ) (Table 4 ). The most common adverse events were "erythra." The one in the treatment group occurred at week 6, presenting as an itchy rash on the scalp that improved after cleaning the skin. Because it improved after skin cleaning, this was considered to be unrelated to the drug and the patient continued drug therapy and this patient was therefore not removed from the study. On the other hand, the adverse event of "erythra" in placebo group occurred at week 4 , with a rash on the surface of limbs accompanied by itching. The symptom disappeared after stopping drug and appeared again when taking drugs. Therefore, the erythra that happened in this patient was considered to have likely been related to the use of hydroxychloroquine. There was one occurrence of pancreatitis, which was considered to be associated with poor primary disease control due to poor medication effectiveness, but the condition was not serious. Only one case of serious adverse events occurred in the placebo group. The patient was found to have abnormal liver function during follow-up at the time of week 4 , which was confirmed as being due to hepatitis B virus (HBV) and therefore unrelated to this study. The three above patients in the placebo group with adverse events were excluded from this study.

\section{Discussion}

Primary Sjögren's syndrome is an incurable chronic disease with long disease duration and frequent flares and can cause 
TABLE 3: Summary of clinical efficacy of pSS patients.

\begin{tabular}{|c|c|c|c|c|c|c|}
\hline & \multicolumn{2}{|c|}{ JieDuTongLuoShengJin granules + HCQ } & \multicolumn{2}{|c|}{ Placebo + HCQ } & \multicolumn{2}{|c|}{$p$ value (between-group) } \\
\hline & Week 4 & Week 12 & Week 4 & Week 12 & Week 4 & Week 12 \\
\hline Dryness $(0-10)$ & & & & & 0.118 & 0.026 \\
\hline Mean \pm SD & $5.1 \pm 2.17$ & $3.6 \pm 2.41$ & $5.6 \pm 2.28$ & $5.2 \pm 2.31$ & & \\
\hline Median (IQR) & $5.0(3.0,7.0)$ & $3.5(1.8,5.3)$ & $6.0(4.8,7.3)$ & $5.5(4.5,7.0)$ & & \\
\hline$p$ value versus week 0 & 0.049 & 0.011 & 0.214 & 0.124 & & \\
\hline Fatigue $(0-10)$ & & & & & 0.765 & 0.002 \\
\hline Mean \pm SD & $5.2 \pm 2.71$ & $3.3 \pm 2.00$ & $5.3 \pm 2.15$ & $4.9 \pm 1.98$ & & \\
\hline Median (IQR) & $5.0(4.0,7.3)$ & $3.0(2.0,5.0)$ & $5.0(4.0,7.0)$ & $5.0(4.0,6.3)$ & & \\
\hline$p$ value versus week 0 & 0.042 & 0.000 & 0.090 & 0.115 & & \\
\hline Pain $(0-10)$ & & & & & 0.853 & 0.322 \\
\hline Mean \pm SD & $2.3 \pm 1.98$ & $1.5 \pm 1.61$ & $2.2 \pm 2.43$ & $1.7 \pm 2.25$ & & \\
\hline Median (IQR) & $2.5(0.0,3.3)$ & $1.0(0.0,2.3)$ & $1.5(0.0,4.0)$ & $0.5(0.0,2.3)$ & & \\
\hline$p$ value versus week 0 & 0.034 & 0.006 & 0.025 & 0.014 & & \\
\hline ESSPRI (0-10) & & & & & 0.217 & 0.002 \\
\hline Mean \pm SD & $4.2 \pm 1.60$ & $2.8 \pm 1.18$ & $4.4 \pm 1.72$ & $3.9 \pm 1.63$ & & \\
\hline Median (IQR) & $4.3(2.7,5.2)$ & $2.7(2,3.7)$ & $4.3(3.7,5.0)$ & $3.9(3,4.8)$ & & \\
\hline$p$ value versus week 0 & 0.009 & 0.000 & 0.039 & 0.032 & & \\
\hline PGA $(0-10)$ & & & & & 0.716 & 0.019 \\
\hline Mean \pm SD & $5.0 \pm 1.75$ & $3.3 \pm 1.68$ & $5.4 \pm 2.03$ & $4.7 \pm 2.01$ & & \\
\hline Median (IQR) & $5.0(3.0,6.3)$ & $3.0(2.0,5.0)$ & $5.0(4.0,7.0)$ & $5.0(3.0,6.0)$ & & \\
\hline$p$ value versus week 0 & 0.008 & 0.000 & 0.042 & 0.016 & & \\
\hline Salivary flow rate $(\mathrm{ml} / 15 \mathrm{~min})$ & & & & & 0.613 & 0.321 \\
\hline Mean \pm SD & $0.8 \pm 0.71$ & $1.1 \pm 0.81$ & $0.7 \pm 0.55$ & $0.9 \pm 0.57$ & & \\
\hline Median (IQR) & $0.9(0.2,1.0)$ & $1.0(0.6,1.5)$ & $0.7(0.3,1.0)$ & $0.9(0.8,1.0)$ & & \\
\hline$p$ value versus week 0 & 0.085 & 0.000 & 0.354 & 0.049 & & \\
\hline Schirmer's test (left) $(\mathrm{mm} / 5 \mathrm{~min})$ & & & & & 0.445 & 0.527 \\
\hline Mean \pm SD & $4.7 \pm 3.89$ & $5.0 \pm 4.44$ & $3.9 \pm 2.78$ & $4.1 \pm 2.44$ & & \\
\hline Median (IQR) & $3.0(2.0,5.3)$ & $4.0(2.0,5.3)$ & $3.0(2.0,5.0)$ & $3.3(2.0,6.0)$ & & \\
\hline$p$ value versus week 0 & 0.457 & 0.284 & 0.938 & 0.585 & & \\
\hline Schirmer's test (right) $(\mathrm{mm} / 5 \mathrm{~min})$ & & & & & 0.543 & 0.759 \\
\hline Mean \pm SD & $4.5 \pm 2.67$ & $5.0 \pm 5.15$ & $3.6 \pm 1.96$ & $4.1 \pm 2.34$ & & \\
\hline Median (IQR) & $4.0(3.0,5.0)$ & $4.0(2.0,6.0)$ & $3.0(2.0,5.0)$ & $4.0(2.0,6.0)$ & & \\
\hline$p$ value versus week 0 & 0.561 & 0.948 & 0.202 & 0.114 & & \\
\hline BUT (left) (second) & & & & & 0.527 & 0.928 \\
\hline Mean \pm SD & $3.7 \pm 1.87$ & $3.9 \pm 1.66$ & $3.0 \pm 1.43$ & $3.6 \pm 1.36$ & & \\
\hline Median (IQR) & $3.5(2.0,4.3)$ & $4.0(2.8,5.0)$ & $2.5(2.0,3.3)$ & $3.0(2.8,4.3)$ & & \\
\hline$p$ value versus week 0 & 0.717 & 0.663 & 0.776 & 0.108 & & \\
\hline BUT (right) (second) & & & & & 0.474 & 0.873 \\
\hline Mean \pm SD & $3.6 \pm 1.38$ & $3.6 \pm 1.39$ & $3.2 \pm 1.15$ & $3.4 \pm 1.50$ & & \\
\hline Median (IQR) & $3.0(3.0,4.0)$ & $3.5(3.0,4.3)$ & $3.0(2.0,4.0)$ & $3.5(2.0,5.0)$ & & \\
\hline$p$ value versus week 0 & 0.490 & 0.479 & 0.763 & 0.299 & & \\
\hline van Bijsterveld (left) (score) & & & & & 0.542 & 0.588 \\
\hline Mean \pm SD & $3.2 \pm 1.50$ & $3.5 \pm 1.93$ & $3.5 \pm 1.67$ & $3.1 \pm 1.80$ & & \\
\hline Median (IQR) & $3.0(2.0,4.0)$ & $3.5(2.0,5.0)$ & $3.0(2.8,4.3)$ & $3.0(1.8,4.3)$ & & \\
\hline$p$ value versus week 0 & 0.460 & 0.877 & 0.720 & 0.769 & & \\
\hline van Bijsterveld (right) (score) & & & & & 0.800 & 0.631 \\
\hline Mean \pm SD & $3.4 \pm 1.60$ & $3.4 \pm 1.90$ & $3.5 \pm 1.67$ & $3.2 \pm 1.76$ & & \\
\hline Median (IQR) & $3.5(2.8,4.0)$ & $3.5(2.0,4.3)$ & $3.5(2.8,4.3)$ & $3.0(2.0,4.3)$ & & \\
\hline$p$ value versus week 0 & 0.482 & 0.456 & 0.550 & 0.919 & & \\
\hline
\end{tabular}


TABle 3: Continued.

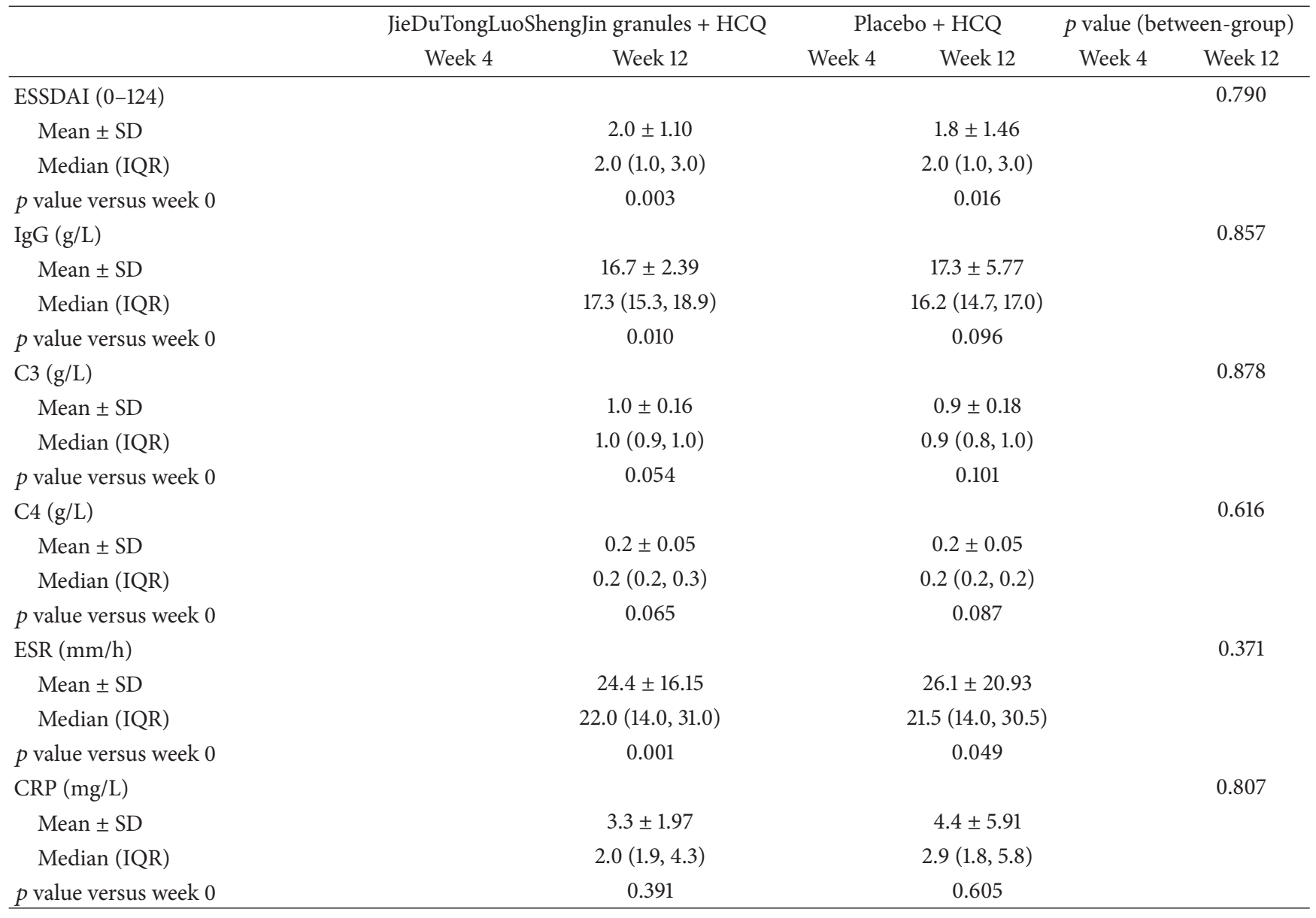

TABLE 4: Analysis of adverse events observed in patients in the treatment groups $(n(\%))$.

\begin{tabular}{lcc}
\hline Event & $\begin{array}{c}\text { JieDuTongLuoShengJin } \\
\text { granules + HCQ (\%) }\end{array}$ & Placebo + HCQ (\%) \\
\hline $\begin{array}{l}\text { Week 4 } \\
\text { Erythra }\end{array}$ & $1(5.0)$ & $0(0.0)$ \\
Week 12 & & \\
Liver dysfunction & $0(0.0)$ & $1(5.0)$ \\
Erythra & $1(5.0)$ & $1(5.0)$ \\
Pancreatitis & $0(0.0)$ & $1(5.0)$ \\
\hline
\end{tabular}

multisystem damage in affected patients. At present, the treatment of Sjögren's syndrome is comprised of traditional immunosuppressants, and one of the most widely used immunosuppressants is hydroxychloroquine (HCQ).

Sjögren's syndrome belongs to the "dryness arthralgia" category in traditional Chinese medicine. Basic Questions first proposed the pathogenesis theory that Yin deficiency can transform into dryness and pointed out the treatment principle of nourishing Yin fluid. Body fluid and blood are derived from a common source, and severe consumption or loss of body fluid will affect the source of blood, leading to stasis of blood. The disease mechanism theory of "drynesstoxicity" was put forward by modern physicians of traditional Chinese medicine, referencing the "toxin factor theory" of epidemic febrile disease. According to this theory, patients suffering from these diseases share common characteristics of stubborn illness, long duration, severe symptomatology, and widespread physical involvement. These are also features of Sjögren's syndrome. Therefore, in traditional Chinese medicine, pSS is induced by "dryness-toxicity" and the blockade of qi and blood, which can lead to static blood and impairment of body fluid, and it results in Yin deficiency. Following these above principles, we set up JieDuTongLuoShengJin, which is a recipe that has been felt to be effective in the bedside treatment of pSS. It contains herbals that nourish Yin and promotes the secretion of fluid, relieves internal heat, promotes blood circulation, and removes obstruction in channels. We used radix paeoniae alba as sovereign drug to replenish qi and nourish blood; rhizoma zedoariae and angelica as minister drugs to strengthen the force of nourishing and invigorating blood circulation; spreading hedyotis herb as assistant drug to help relieve internal heat; Astragalus 
membranaceus as contrary drug to invigorate qi and promote circulation of blood; licorice root as an envoy drug to harmonize all of the above drugs. All the drugs were mixed together with the effect of detoxification, regulating the meridians, and promoting the secretion of saliva or body fluid.

Modern pharmacological evidence suggests that the total glycosides of paeoniae extracts from radix paeoniae alba could reduce serum immunoglobulin and erythrocyte sedimentation rate, improve symptoms such as xerophthalmia and xerostomia [24], and have bidirectional regulation effects on the proliferation of $\mathrm{T}$ and $\mathrm{B}$ lymphocytes to some extent, promoting or inhibiting the production of interleukin 1, interleukin 2, and tumor necrosis factor [25]. In previous experiments using nonobese diabetic mice, spreading hedyotis herb has been shown to improve body weight, water intake, salivary flow rate, and submandibular gland index [26]. Furthermore, it raises the expression of AQP5mRNA in salivary gland and submandibular gland tissue to some extent and this and additional medications may be able to increase the amount of AQP5, which can spur moisture transport rate, increase a cell's cytoplasmic volume, promote gland secretion, and therefore improve the symptoms of xerostomia [27, 28].

Previous research using JieDuTongLuoShengJin granules has demonstrated reduced immune globulin and the submandibular gland index in rat models of Sjögren's syndrome [29, 30]. The above evidence suggests that JieDuTongLuoShengJin is a very good immune regulator.

Our data in early clinical studies reveals that JieDuTongLuoShengJin use can improve some subjective symptoms and objective indicators in pSS [11]. This clinical trial aimed to verify those findings using a randomized, placebo-controlled, double-blind method. Patients with mild disease activity and without visceral involvement of pSS received oral JieDuTongLuoShengJin granules twice daily, or placebo granules, both in combination with a stable dose of HCQ.

In order to evaluate disease more comprehensively, we chose both the EULAR SS patient-reported index (ESSPRI) (the primary endpoint) and the EULAR SS disease activity index (ESSDAI) (the most important secondary endpoint) as the index of subjective symptoms and systemic features, two complementary ways to quantitatively measure disease severity that have been shown to be valid and sensitive measures [31,32]. The ESSPRI score correlates well with other measures such as Patient Global Assessment (PGA), Profile of Fatigue and Discomfort (PROFAD) [33], and Sicca Symptoms Inventory (SSI) [34]. The ESSDAI score has better sensitivity [15] compared to other scales such as Sjögren's Syndrome Disease Activity Index (SSDAI) [35] and Sjögren's Systemic Clinical Activity Index (SCAI) [36]. There is no significant correlation between the two indices, but both are sensitive to changes in disease activity [37]. This study demonstrates that JieDuTongLuoShengJin, a traditional Chinese medicine, is overall superior to placebo in improving patients' subjective symptoms using the ESSPRI score $(p<0.01)$ and that each of the domains within the ESSPRI score decreased observably after treatment $(p<0.05)$. More than three-quarters of patients achieved MCII in the treatment group, significantly higher than the control group $(p<0.05)$. JieDuTongLuoShengJin granules + HCQ treatment also showed significant
PGA improvement $(p=0.008$ at week 4 and $p=0.000$ at week 12), and although there was improvement in the ESSDAI score at week $12(p=0.003)$, it was not obviously different from that in the placebo group $(p=0.790)$. Therefore the combination of JieDuTongLuoShengJin granules with HCQ is superior to placebo combined with HCQ in improving subjective pSS symptoms, but not in modifying the degree of disease activity.

Furthermore, this study revealed that the levels of serum ESR diminished significantly in JieDuTongLuoShengJin + HCQ group. There was also an improvement in unstimulated saliva flow rate and reduced serum IgG, but this was not significantly different from that in the placebo group. Furthermore, neither treatment nor placebo groups showed CRP improvement. These results were not completely consistent with previous related studies, which have shown that HCQ can improve patient's fatigue and joint symptoms, and effectively reduce immunoglobulin, ESR, and CRP levels $[38,39]$. In our present study, we chose the patients with low-activity-level pSS (ESSDAI < 5) but without visceral involvement, and our observation period was relatively brief. This may partly explain why our results were different from those of previous studies. In addition, in previous clinical studies, HCQ has been identified in patients with pSS as having a role of decreasing the level of $\mathrm{B}$ cell activating factor belonging to the TNF family (BAFF) in the serum and glands, reducing the concentration of cholinesterase, triggering the increasing of stimulated saliva flow rates and tear secretion, and finally improving sicca symptoms [40-43]. Animal experiments [44] indicate that HCQ can obviously downregulate the expression of interleukin 1 beta (IL-1 $\beta$ ) and tumor necrosis factor alpha (TNF- $\alpha$ ) in local submandibular gland of nonobese diabetic (NOD) mice on the levels of gene transcription and protein synthesis. It can reduce the content of IL- $1 \beta$ and TNF- $\alpha$ in peripheral blood serum, increase saliva flow rate, and reduce submandibular gland tissue damage on pathological examination.

This study demonstrates that although dryness symptoms and salivary flow rate improved with treatment, the effect on ocular dryness was not dramatic in either group. There were no significant differences of Schirmer's test, BUT, and ocular surface damage score before and after treatment in both groups, similar to previous research by Akpek et al. [45]. It is possible that these objective features vary modestly over time and therefore did not clearly exhibit a sufficient sensitivity to change [46-49]. For this reason, these measures have not been emphasized in recent trials $[23,50]$.

It is worth noting that similar proportions of (JieDuTongLuoShengJin granules + HCQ)-treated and (placebo + HCQ)-treated patients reported adverse event (AEs) during the study, and half of these were unlikely to have been related to the treatments those patients received: one had erythra that was felt to be unrelated to the study, and the other had hepatitis B virus infection. JieDuTongLuoShengJin granules are therefore safe in clinical use.

In conclusion, when combined with HCQ as a treatment, JieDuTongLuoShengJin granules show good effect in improving the subjective symptoms and some objective indicators of pSS. These results indicate that combination with 
JieDuTongLuoShengJin granules is promising for relieving symptoms and helpful to patients overall. It is a safe and effective treatment strategy for patients with pSS and deserves further clinical and experimental research.

\section{Conflicts of Interest}

The authors declare that they have no conflicts of interest.

\section{Acknowledgments}

This work was supported by Shanghai Municipal Commission of Health and Family Planning (Project no. 2014LZ107A) and National Natural Science Foundation of China (Project no. 81573925$)$.

\section{References}

[1] J. M. Meijer, P. M. Meiners, J. J. R. H. Slater et al., "Healthrelated quality of life, employment and disability in patients with sjögren's syndrome," Rheumatology, vol. 48, no. 9, pp. 10771082, 2009.

[2] C. G. Helmick, D. T. Felson, R. C. Lawrence et al., "Estimates of the prevalence of arthritis and other rheumatic conditions in the United States. Part I," Arthritis \& Rheumatism, vol. 58, no. 1, pp. 15-25, 2008.

[3] F. Wang, Z. G. Wang, and F. L. Tang, "Expression of chemokine in the labial glands of patients with Sjogren's syndrome (in Chinese)," National Medical Journal of China, vol. 83, no. 3, pp. 220-221, 2003.

[4] L. T. H. Jacobsson, T. E. Axell, B. U. Hansen et al., "Dry eyes or mouth-an epidemiological study in Swedish adults, with special reference to primary Sjögren's syndrome," Journal of Autoimmunity, vol. 2, no. 4, pp. 521-527, 1989.

[5] V. Devauchelle-Pensec, X. Mariette, S. Jousse-Joulin et al., "Treatment of primary Sjögren syndrome with rituximab: a randomized trial," Annals of Internal Medicine, vol. 160, no. 4, pp. 233-242, 2014.

[6] S. Bowman, C. Everett, and M. Bombardieri, "Preliminary Results of a Double-Blind Randomised Trial of Rituximab AntiB-Cell Therapy in Patients with Primary Sjogrens Syndrome," Arthritis Rheumatol, vol. 67, supplement 10, 2015.

[7] S. D. Vita, L. Quartuccio, R. Seror et al., "Efficacy and safety of belimumab given for 12 months in primary Sjögren's syndrome: the BELISS open-label phase II study," Rheumatology, vol. 54, no. 12, pp. 2249-2256, 2016.

[8] M. Ramos-Casals and J. Font, "Primary Sjögren syndrome," in Current Diagnosis and Treatment in Rheumatology, J. Imboden, D. Hellmann, and J. Stone, Eds., vol. 304, McGraw-Hill, New York, NY, USA, 2007.

[9] H. Luo, X. Li, J. Liu, F. Andrew, and L. George, "Chinese herbal medicine in treating primary Sjögren's syndrome: a systematic review of randomized trials," Evidence-based Complementary and Alternative Medicine, vol. 2012, Article ID 640658, pp. 1-22, 2012.

[10] M. Ramos-Casals, G. A. Tzioufas, H. J. Stone, A. Sisó, and X. Bosch, "Treatment of primary Sjögren syndrome: a systematic review," Journal of the American Medical Association, vol. 304, no. 4, pp. 452-460, 2010.

[11] H. Jian-dong, L. Guo-ling, and X. Luan, "The efficacy and safety of the therapeutic method of detoxicating, relieving meridian and engendering fluid in the treatment of the primary sjogren syndrome," Chinese Jourmal of Basic Medicine in Traditional Chinese Medicine, vol. 19, no. 1, pp. 70-73, 2013.

[12] W. Hu, X. Qian, F. Guo et al., "Traditional Chinese medicine compound ShengJinRunZaoYangXue granules for treatment of primary Sjögren's syndrome: a randomized, double-blind, placebo-controlled clinical trial," Chinese Medical Journal, vol. 127, no. 15, pp. 2721-2726, 2014.

[13] C. Vitali, S. Bombardieri, R. Jonsson et al., "Classification criteria for Sjögren's syndrome: a revised version of the European criteria proposed by the American-European consensus group," Annals of the Rheumatic Diseases, vol. 61, no. 6, pp. 554-558, 2002.

[14] R. Seror, P. Ravaud, X. Mariette et al., "EULAR Sjögren's Syndrome Patient Reported Index (ESSPRI): development of a consensus patient index for primary Sjögren's syndrome," Annals of the Rheumatic Diseases, vol. 70, no. 6, pp. 968-972, 2011.

[15] R. Seror, P. Ravaud, S. J. Bowman et al., "EULAR Sjögren's syndrome disease activity index: development of a consensus systemic disease activity index for primary Sjögren's syndrome," Annals of the Rheumatic Diseases, vol. 69, no. 6, pp. 1103-1109, 2010.

[16] R. Seror, H. Bootsma, A. Saraux et al., "Defining disease activity states and clinically meaningful improvement in primary Sjögren's syndrome with EULAR primary Sjögren's syndrome disease activity (ESSDAI) and patient-reported indexes (ESSPRI)," Annals of the Rheumatic Diseases, vol. 75, no. 2, pp. $1-8,2014$.

[17] C. Dawes, "Circadian rhythms in human salivary flow rate and composition," The Journal of Physiology, vol. 220, no. 3, pp. 529$545,1972$.

[18] D. B. Ferguson, A. Fort, A. L. Elliott, and A. J. Potts, "Circadian rhythms in human parotid saliva flow rate and composition," Archives of Oral Biology, vol. 18, no. 9, pp. 1155-1173, 1973.

[19] F. R. Burlage, J. Pijpe, R. P. Coppes et al., "Variability of flow rate when collecting stimulated human parotid saliva," European Journal of Oral Sciences, vol. 113, no. 5, pp. 386-390, 2005.

[20] W. W. Kalk, K. Mansour, A. Vissink et al., "Oral and ocular manifestations in Sjögren's syndrome," Journal of Rheumatology, vol. 29, no. 5, pp. 924-930, 2002.

[21] O. P. van Bijsterveld, "Diagnostic tests in the Sicca syndrome," Archives of Ophthalmology, vol. 82, no. 1, pp. 10-14, 1969.

[22] R. Jonsson, P. Vogelsang, R. Volchenkov, A. Espinosa, M. Wahren-Herlenius, and S. Appel, "The complexity of Sjögren's syndrome: Novel aspects on pathogenesis," Immunology Letters, vol. 141, no. 1, pp. 1-9, 2011.

[23] J. M. Meijer, P. M. Meiners, A. Vissink et al., "Effectiveness of rituximab treatment in primary sjögren's syndrome: a randomized, double-blind, placebo-controlled trial," Arthritis and Rheumatism, vol. 62, no. 4, pp. 960-968, 2010.

[24] H. Zhao and F.-T. Zhao, "Clinical observation on effect of total glucosides of paeony combined with hcq in treating patients with primary sjögren's syndrome," Henan Traditional Chinese Medicine, vol. 33, no. 4, pp. 530-531, 2013 (Chinese).

[25] H.-F. Zhang, P. Hou, and Xiao W.-G., "Clinical observation on effect of total glucosides of paeony in treating patients with nonsystemic involved sjögren syndrome," CJITWM, vol. 27, no. 7, pp. 596-598, 2007 (Chinese).

[26] G.-F. Hao, C.-P. Wen, and Y.-S. Li, "Experimental research of the treatments of heat -clearing and detoxication for sicca 
syndrome," Chinese Archives of Traditional Chinese Medicine, vol. 30, no. 9, article 2125, 2012.

[27] W.-K. Ma, E.-T. Jia, L.-M. Liu et al., "Gene expression of AQP1, AQP5 in Sjogren's syndrome model mice salivary gland, and the therapeutic effect of jiedu huayu herbs," New Journal of Traditional Chinese Medicine, vol. 43, no. 10, article 111, 2011 (Chinese).

[28] W.-K. Ma, Y. Wang, F. Tang et al., "Regulation function of Jiedu Huayu prescriptions for channel water molecules AQP5 protein in the submandibular gland of sjogren's syndrome model mice," Chinese Journal of Gerontology, vol. 32, no. 1, p. 95, 2012.

[29] D. Wang, H. Zhao, and L. Qiuju, "Analysis of the expression of AQP5 in rats with Sjogren syndrome intervened by Detoxicating Medicine, Meridians-relieving Medicine and Engender Fluid Medicine," China Medical Herald, vol. 9, no. 19, p. 26, 2012.

[30] D. Wang, X. Luan, and L. Qiuju, “The preliminary research about the efficacy of the therapeutic method of Detoxicating, Relieving Meridian and Engendering Fluid in sjogren's syndrome rats on the immune function," Zhejiang Journal of Traditional Chinese Medicine, vol. 46, no. 11, pp. 837-839, 2011.

[31] R. Seror, E. Theander, J. G. Brun et al., "Validation of EULAR primary Sjögren's syndrome disease activity (ESSDAI) and Patient indexes (ESSPRI)," Annals of the Rheumatic Diseases, vol. 74, no. 5, pp. 859-866, 2015.

[32] R. Seror, X. Mariette, and S. Bowman, "Accurate detection of changes in disease activity in primary sjogren's syndrome by the european league against rheumatism Sjogren's syndrome disease activity index," Arthritis Care \& Research, vol. 62, no. 4, pp. 551-558, 2010.

[33] S. J. Bowman, D. A. Booth, R. G. Platts et al., "Measurement of fatigue and discomfort in primary Sjögren's syndrome using a new questionnaire tool," Rheumatology, vol. 43, no. 6, pp. 758764, 2004.

[34] S. J. Bowman, D. A. Booth, R. G. Platts, A. Field, and J. Rostron, "Validation of the Sicca Symptoms Inventory for clinical studies of Sjögren's syndrome," Journal of Rheumatology, vol. 30, no. 6, pp. 1259-1266, 2003.

[35] C. Vitali, G. Palombi, C. Baldini et al., "Sjögren’s syndrome disease damage index and disease activity index: scoring systems for the assessment of disease damage and disease activity in Sjögren's syndrome, derived from an analysis of a cohort of Italian patients," Arthritis and Rheumatism, vol. 56, no. 7, pp. 2223-2231, 2007.

[36] S. J. Bowman, N. Sutcliffe, D. A. Isenberg et al., "Sjögren's Systemic Clinical Activity Index (SCAI) - a systemic disease activity measure for use in clinical trials in primary Sjögren's syndrome," Rheumatology, vol. 46, no. 12, pp. 1845-1851, 2007.

[37] P. M. Meiners, S. Arends, E. Brouwer, F. K. L. Spijkervet, A. Vissink, and H. Bootsma, "Responsiveness of disease activity indices ESSPRI and ESSDAI in patients with primary Sjögren's syndrome treated with rituximab," Annals of the Rheumatic Diseases, vol. 71, no. 8, pp. 1297-1302, 2012.

[38] S.-Q. Shuai and H.-B. Sun, “Treatment of primary Sjogren's syndrome with hydroxychloroquine," West China Medical Journal, vol. 23, no. 7, pp. 1281-1283, 2011.

[39] H. Hao, "Clinical observation of sjogren's syndrome with the treatment of Radix paeoniae alba total glycosides in combination with hydroxychloroquine," Hubei journal of traditional Chinese medicine, vol. 32, no. 11, pp. 30-31, 2010.

[40] G. Mumcu, M. Biçakçigil, N. Yilmaz et al., "Salivary and serum B-cell activating factor (BAFF) levels after hydroxychloroquine treatment in primary Sjögren's syndrome," Oral health \& preventive dentistry, vol. 11, no. 3, pp. 229-234, 2013.

[41] S. Yavuz, E. Asfuroğlu, M. Bicakcigil, and E. Toker, "Hydroxychloroquine improves dry eye symptoms of patients with primary Sjogren's syndrome," Rheumatology International, vol. 31, no. 8, pp. 1045-1049, 2011.

[42] M. Rihl, K. Ulbricht, R. E. Schmidt, and T. Witte, "Treatment of sicca symptoms with hydroxychloroquine in patients with Sjögren's syndrome," Rheumatology, vol. 48, no. 7, pp. 796-799, 2009.

[43] H. Cankaya, E. Alpöz, G. Karabulut, P. Güneri, H. Boyacoglu, and Y. Kabasakal, "Effects of hydroxychloroquine on salivary flow rates and oral complaints of Sigren patients: a prospective sample study," Oral Surgery, Oral Medicine, Oral Pathology, Oral Radiology and Endodontology, vol. 110, no. 1, pp. 62-67, 2010.

[44] J.-Q. Cao, L.-J. Zhao, W.-G. XIao et al., "Effect of Hydroxychlorquine on IL-1 $\beta$ and TNF- $\alpha$ in non-obese diabetic mice with Sjögren's syndrome," Chinese Journal of Rheumatology, vol. 10, no. 9, pp. 542-546, 2006 (Chinese).

[45] E. K. Akpek, K. B. Lindsley, R. S. Adyanthaya, R. Swamy, A. N. Baer, and P. J. McDonnell, "Treatment of Sjögren's syndromeassociated dry eye. An evidence-based review," Ophthalmology, vol. 118, no. 7, pp. 1242-1252, 2011.

[46] F. B. Vivino, I. Al-Hashimi, Z. Khan et al., "Pilocarpine tablets for the treatment of dry mouth and dry eye symptoms in patients with Sjogren syndrome: a randomized, placebocontrolled, fixed- dose, multicenter trial," Archives of Internal Medicine, vol. 159, no. 2, pp. 174-181, 1999.

[47] D. Petrone, J. J. Condemi, R. Fife, O. Gluck, S. Cohen, and P. Dalgin, "A double-blind, randomized, placebo-controlled study of cevimeline in Sjögren's syndrome patients with xerostomia and keratoconjunctivitis sicca," Arthritis and Rheumatism, vol. 46, no. 3, pp. 748-754, 2002.

[48] G. Gannot, H. E. Lancaster, and P. C. Fox, "Clinical course of primary Sjögren's syndrome: salivary, oral, and serologic aspects," The Journal of Rheumatology, vol. 27, no. 8, pp. 19051909, 2000.

[49] B. K. S. Davidson, C. A. Kelly, and I. D. Griffiths, "Primary Sjogren's syndrome in the North East of England: a long-term follow-up study," Rheumatology, vol. 38, no. 3, pp. 245-253, 1999.

[50] J. Pijpe, G. W. Van Imhoff, F. K. L. Spijkervet et al., "Rituximab treatment in patients with primary Sjögren's syndrome: an open-label phase II study," Arthritis and Rheumatism, vol. 52, no. 9, pp. 2740-2750, 2005. 


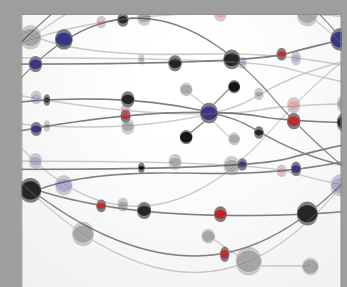

The Scientific World Journal
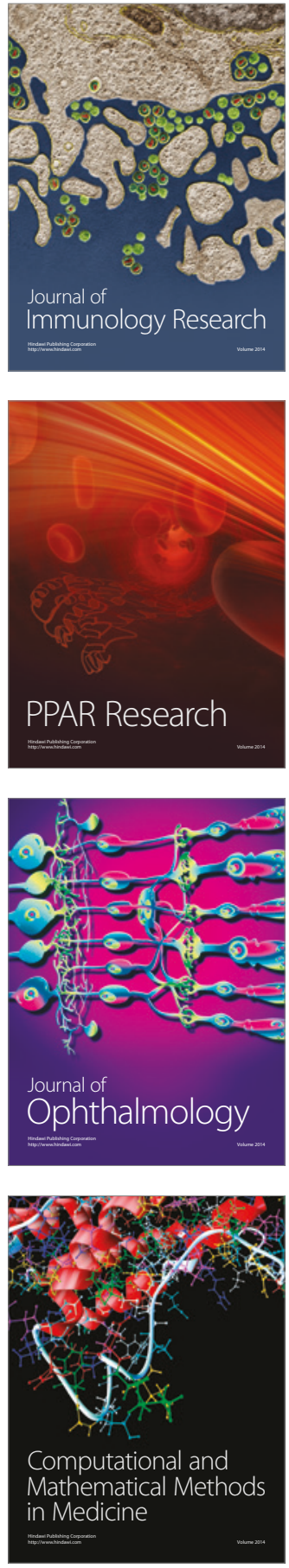

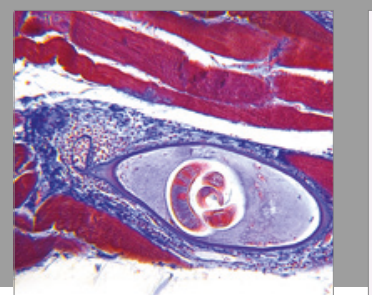

Gastroenterology Research and Practice
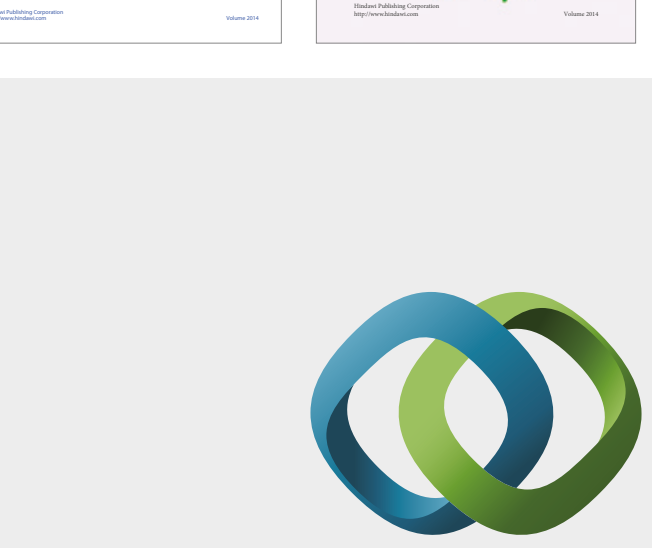

\section{Hindawi}

Submit your manuscripts at

https://www.hindawi.com
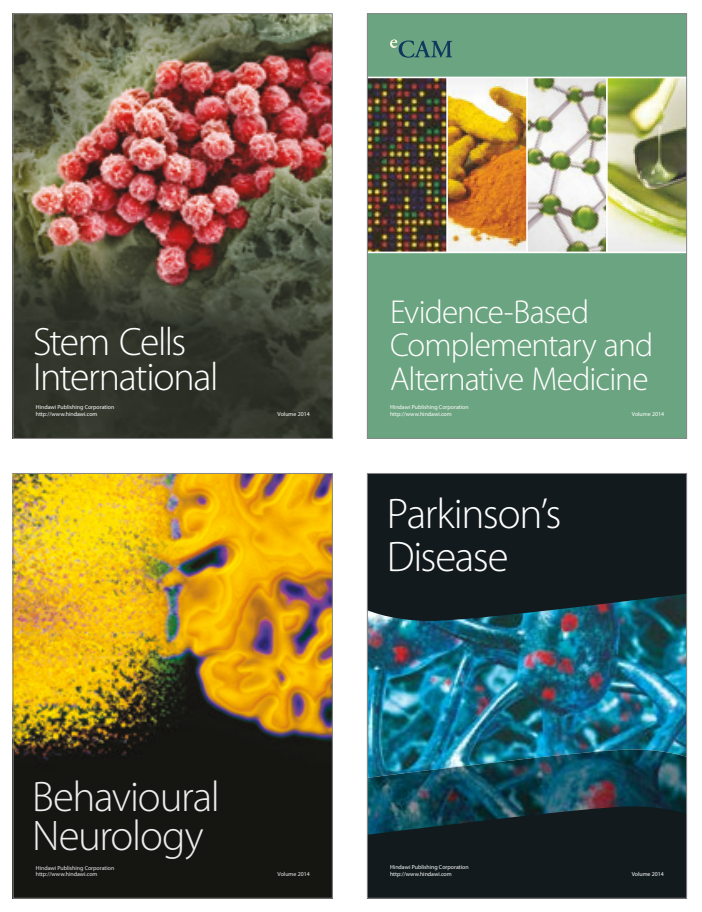
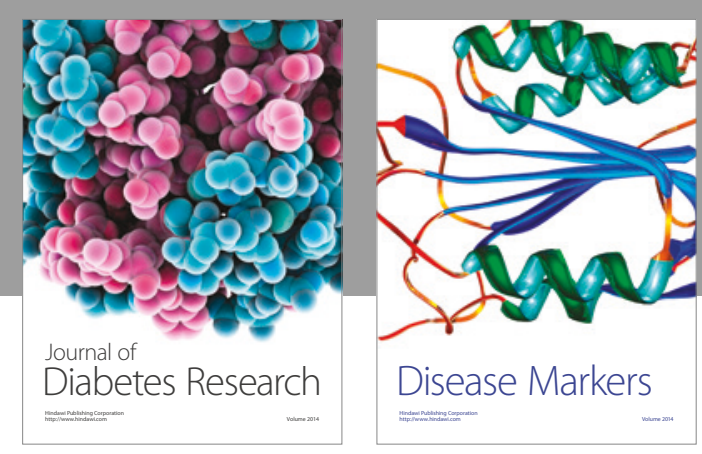

Disease Markers
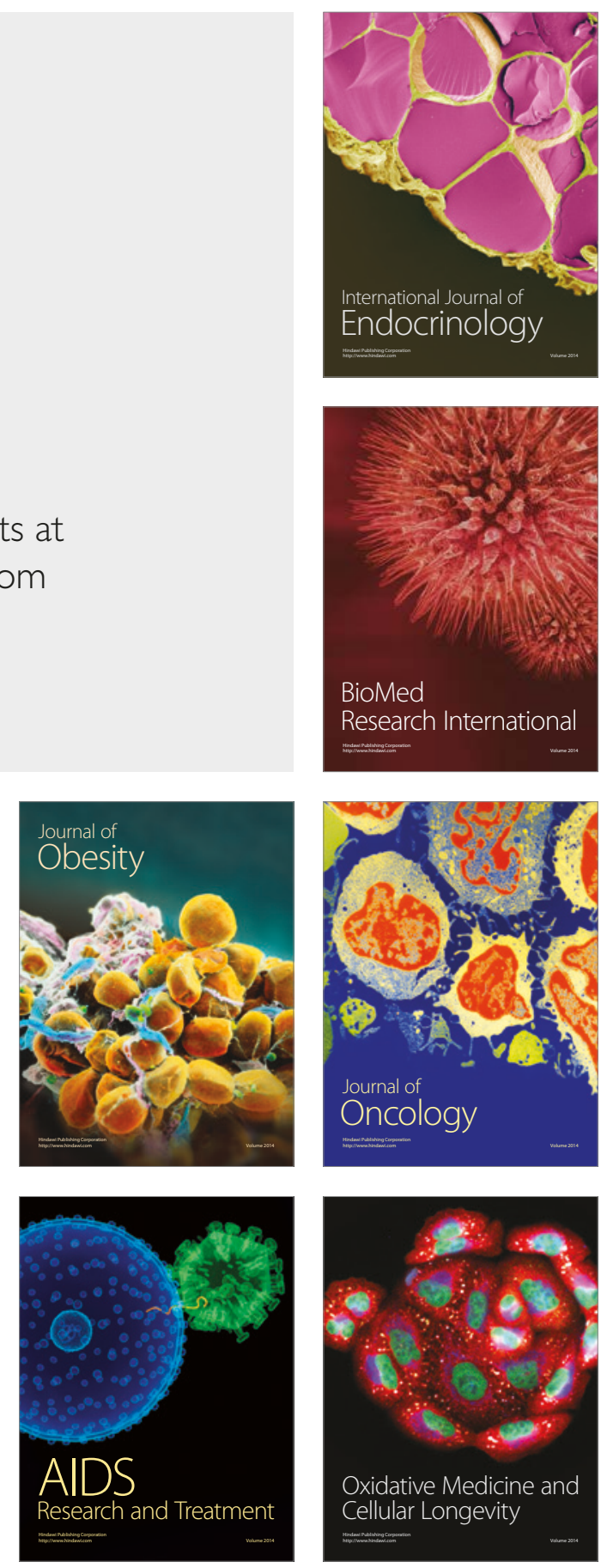